\title{
Asedio, memoria y justicia: los fantasmas de Trujillo
}

Siege, Memory and Justice: The Ghosts of Trujillo

Carlos Andrés Ramírez Arenas ${ }^{\star}$ Universidad de los Andes-Colombia

Recibido: 03 de marzo de 2014

Enviado a pares: 03 de marzo de 2014

Aceptado por pares: 24 de agosto de 2014

Aprobado: 04 de septiembre de 2014

Pensamiento y Cultura | ISSN: 0123-0999 | eISSN: 2027-5331

pensam.cult | Vol. 17-2 | Diciembre de 2014 | pp. 118-144

DOI: 10.5294/pecu.2014.17.2.6 


\section{Asedio, memoria y justicia: los fantasmas de Trujillo}

Resumen: El siguiente artículo reflexiona sobre la responsabilidad que tenemos actualmente los pensadores y científicos sociales en la tarea de construcción y crítica de la memoria histórica. El artículo toma como marco teórico las reflexiones de Jacques Derrida en torno al 'fantasma' y a su comprensión de la justicia como una forma de responsabilidad que va más allá de las formas de justicia presentes en el derecho. Además, toma las labores, reclamos y prácticas de la Asociación de Familiares de Víctimas de Trujillo (AFAVIT) como un ejemplo actual de cómo la memoria de los fantasmas es la memoria que posibilita esta otra forma de justicia.

Palabras clave: fantasma, historia, memoria, justicia, responsabilidad, Trujillo.

\section{Siege, Memory and Justice: The Ghosts of Trujillo}

Abstract: The following paper reflects on the responsibility that we, thinkers and social scientists, currently have in the task of building and criticizing historic memory. The paper takes as its theoretical framework Jacques Derrida's reflections with regard to the 'ghost', and his comprehension of justice as a form of responsibility that exceeds the forms of justice allowed by law. Moreover, we take the labors, claims and practices of the 'Asociación de Familiares de Víctimas de Trujillo' (AFAVIT) as an example of how the memory of ghosts is the memory that enables this other form of justice.

Key words: Ghost, history, memory, justice, responsibility, Trujillo. 
El pasado lleva consigo un secreto índice, por el cual es remitido a la redención.

¿Acaso no nos roza un hálito del aire que envolvió a los precedentes? [...] Si es así, entonces existe un secreto acuerdo entre las generaciones pasadas y la nuestra. Entonces hemos sido esperados en la tierra. Entonces nos ha sido dada, tal como a cada generación que nos precedió, una débil fuerza mesiánica, sobre la cual el pasado reclama derecho. No es fácil atender a esta reclamación. Walter Benjamin, Tesis II, Sobre el concepto de historia

No es lugar de muertos, es jardín de vivos, olor a resistencia, es memoria histórica, denuncia subversiva, es duelo doloroso de llanto y sufrimiento, es sueño y utopía de nueva sociedad Asociación de Familiares de Víctimas de Trujillo, 'Parque-Monumento'

En nuestro contexto colombiano - así como en otros países de Latinoamérica- las discusiones sobre la memoria nacieron no de una mirada pretendidamente desinteresada o meramente curiosa al pasado, sino de una situación violenta y dolorosa - muchas veces asumida solo como pasada ${ }^{1}$ - que exigía, de cierta manera, una postura crítica frente a las narrativas hegemónicas relativas a la memoria de lo acontecido. Todo esto con el fin de que, de una u otra manera, se hiciera justicia con el pasado y con las víctimas de los conflictos y la violencia. Los ejercicios de memoria histórica asumen entonces cierta responsabilidad ética y política en la medida en que buscan contribuir a que se haga justicia con los eventos y las víctimas del pasado y que resuenan aún en el presente. En medio de esta necesidad por construir historia y memoria ${ }^{2}$,

1 Con respecto a cómo ciertos eventos del pasado no pueden pensarse como 'pasados' sino como supervivientes y presentes en nuestra experiencia cotidiana, es interesante y sugestivo el texto de Veena Das, Life and Words. En una medida importante, el enfoque que le daremos a nuestra lectura del texto de Derrida está influenciado por algunas de las ideas que Das expone en su trabajo.

2 Veremos que las maneras en que memoria e historia puedan ser construidas son muy variadas. Sin embargo, será importante en este texto mostrar de qué manera cada ejercicio 
no solo hemos visto cómo se ha dado una proliferación de discursos que buscan construirla (crearla, producirla) y establecer una posición crítica respecto a cómo se debería concebir esa construcción, sino que dicha necesidad se ha constituido como un problema ineludible para la academia, el Estado, el arte y otras perspectivas y espacios de la sociedad colombiana. Ante dicha situación de asedio de memoria, no solo de los eventos traumáticos del pasado sino además de la necesidad misma de tener que responder de alguna manera a ellos, pensamos que la postura de Derrida tiene valiosos elementos para aportarnos.

Jacques Derrida en su conferencia Espectros de Marx reflexiona sobre cómo es posible ser herederos de Marx, o cómo es posible pensar una herencia crítica del marxismo, en un tiempo en el cual Marx había sido observado con recelo e incluso había llegado a ser declarado como un pensador en desuso. A través de esta reflexión, Derrida aborda temas que exceden la pregunta - aunque no carentes de complejidad- por el lugar de Marx en el tardío siglo XX, como la herencia, el duelo, la justicia, el tiempo, entre otros. Todos estos temas y nociones están mediados, o condicionados, por la noción de lo que Derrida llama lo espectral o lo fantasmal.

Ahora bien, podemos intuir, por el momento, un primer aspecto de lo fantasmal en esta forma de relacionarnos con el pasado que se presenta actualmente: este pasado que vuelve constantemente, que no parece poder olvidarse y que reclama sobre sí una atención continua que, en gran medida, implica exponerse a un terror y un dolor no fáciles de confrontar. Este asedio nos sobreviene de manera involuntaria pues no parece ser tan siquiera una posibilidad hacer caso omiso a ese pasado ni pensar que de alguna forma sea indiferente, o que en el trabajo de memoria histórica no haya nada que sea necesario producir. Hay una cierta obligación a hablar de ese pasado, pues nuestro presente lo reclama insistentemente. Ante esta experiencia colectiva no podemos sentirnos extrañados: la manera en que cada uno de nosotros recuerda eventos de su propia vida, no se diferencia sustancialmente de esta situación

de memoria asume cierta forma de responsabilidad con el pasado. Veremos, además, que algunas de ellas, por diversas razones, merecen ser sometidas a crítica. 
actual. Hay recuerdos, imágenes y experiencias del pasado que vuelven a nuestra conciencia y que se expresan también en diversos ámbitos de nuestra vida cotidiana sin que podamos controlarlos ni decidir cuándo recordarlos. Se trata, en cierta medida, de una experiencia inconsciente de la memoria, pues el pasado - invertido el esquema habitual- se apodera de nosotros, nos obsesiona, nos asedia, no nos deja en paz y, aunque perteneciente a otro tiempo - en teoría superado-, se 'integra' plenamente a nuestra vida. En ese sentido, la postura de Derrida nos brinda luces sobre cómo entender ese carácter ineludible del pasado y, a la vez, cómo responderle o asumirlo en tanto que se nos presenta con una urgencia inaplazable.

Así pues, en un primer momento del texto elaboraremos una exposición de lo que busca explorar Derrida con estos conceptos, tomando aquello que consideramos es preciso ${ }^{3}$ de ellos y por eso, significativo. En un segundo momento del texto, trataremos de explorar un ejemplo de cómo en las secuelas de un evento histórico tan terrible como la masacre de Trujillo, se han erigido diversas formas de narración y de memoria que dan cuenta de una manera específica de asumir esa responsabilidad con el pasado y, de paso, una cierta comprensión de lo que sería 'hacerle justicia. En particular haremos referencia a algunos de los trabajos, discursos y prácticas que lleva a cabo la Asociación de Familiares de Víctimas de Trujillo (AFAVIT) ${ }^{4}$ apoyándonos también en el informe

3 Derrida hace bastante énfasis en que aquello que es preciso - lo que se hace necesariodebe ser pensado de la mano con lo fantasmal. Alguna indicación a este respecto haremos con la debida precisión en el texto, pero hemos de notar que el ejercicio de interpretación de un escrito debe responder a aquello que se hace necesario en su lectura. Es decir, nuestra selección, énfasis e interés - nuestro afán de interpretación- debe entenderse como aquello que nos ha llamado a pensar algo propio de nuestro presente. La interpretación de un texto debe entenderse, entonces, como lo que surge ante la urgencia de un pensamiento.

4 La AFAVIT lucha para que los 300 casos de violaciones a los derechos humanos en los municipios de Trujillo, Riofrío y Bolívar del Valle del Cauca en Colombia, entre los años de 1987 y 1994, no queden en la impunidad. Su trabajo, por supuesto, se caracteriza por asegurarse de que el Estado colombiano imparta justicia con respecto a las masacres y los homicidios cometidos en esos municipios. Sin embargo, como trataremos de ver más adelante, esta Asociación también pone de manifiesto una serie de preocupaciones a través de ciertos discursos y prácticas que, por su parte, también anhelan una cierta forma de justicia, pero que son incompatibles con lo que permite y promete la ley. 
del Centro Nacional de Memoria Histórica, Trujillo: Una tragedia que no cesa. Con respecto a estos casos, nos enfocaremos en la experiencia de asedio de un pasado que nos reclama a nosotros, los vivos, tener la mirada puesta sobre él.

\section{Asedio y fantasma}

¿Qué es lo fantasmal y por qué resulta significativo para nuestra experiencia cotidiana, más allá de la pregunta específica - para Derrida absolutamente necesaria ${ }^{5}$ - por la actualidad del marxismo? Hay por lo menos dos elementos que a nuestro juicio resultan imprescindibles y altamente sugestivos: por un lado, su forma de asedio - en tanto que hostiga, acosa, insiste - y, por el otro, su acuciamiento - su carácter urgente, inaplazable, incontrolable-.

El fantasma es el que (o la que, los que, lo que) se nos presenta constantemente aunque no queramos tener nada que ver con él. El asedio es, por ello, insistente y a la vez anacrónico. Insistente pues se rehúsa a desaparecer y anacrónico en tanto que el fantasma es alguien ${ }^{6}$ que, se supone, ya no debería estar y que sin embargo parece 'seguir vivo'. La aparición del fantasma trasciende así las estructuras temporales cotidianas de nuestra experiencia, puesto que se resiste al paso del tiempo - no se desgasta sino que conserva su 'vitalidad' - y se encuentra en un intervalo que desestabiliza el orden y la progresión 'natural' de los tiempos: del pasado, al presente y luego al futuro. El fantasma, al cual consideramos perteneciente al pasado, se actualiza en nuestra experiencia y a la vez anuncia su siempre inminente pero imprevisto por-venir. No es nunca el que aparece una vez y no lo vuelve hacer - una simple ilusión

5 A pesar de este 'más allá de la pregunta específica por el marxismo, pensamos que lo que Derrida tiene en mente al reflexionar sobre la actualidad del marxismo tiene que ver con cierto espíritu crítico que no siempre tiene que estar acompañado por el nombre propio de Marx. Es decir - como lo menciona el propio Derrida- la herencia de Marx consiste en algo más que simplemente conocer una doctrina o atenerse a un programa políticorevolucionario: se trata de cómo interpretamos a Marx de manera que podamos tomar de él un impulso crítico para cuestiones que importan en el presente.

6 En todo caso, nunca una entidad, nunca una identidad, nunca un sujeto definido ni definible. 
o engaño-, sino aquel que se ha presentado varias veces y quien parece poder volver a aparecer. Reúne en su presencia - que es también una no-presencia- diversos tiempos. Su aparición no es reductible a una simple 'ilusión' sino a una presencia - medio-presencia- que tiene, sin duda, una forma de 'realidad' sin que esté — 'propiamente' hablando'realmente' allí. Es por esto que el fantasma tiene una relación de asedio con los vivos, pues aparece siempre intempestivamente y sin que estos tengan ningún control o poder de decisión sobre su presentación.

Derrida afirma por ello que la presencia espectral "nos mira y nos ve no verla incluso cuando está ahí" (2012, 21). Cuando el fantasma está ahí, no podemos realmente afirmar que lo veamos, pues su forma de presencia es ambivalente y confusa, pero sí nos sentimos ante $s u$ presencia y ante $s u$ mirada. Este espectro del pasado se abalanza sobre nosotros sin que podamos hacer nada al respecto. No podemos ante el fantasma, no tenemos ningún poder ante él: "[E]ste algún otro espectral nos mira, nos sentimos mirados por él, fuera de toda sincronía, antes incluso y más allá de toda mirada por nuestra parte, conforme a una anterioridad [...] y a una disimetría absolutas, conforme a una desproporción absolutamente indominable" (Derrida 2012, 21). En este primer sentido ${ }^{7}$ la memoria de lo fantasmal sería una memoria involuntaria e impersonal pues ella se impone autoritaria y violentamente. Lo que hagamos o sintamos que debemos hacer ante esa presencia es algo de lo que hablaremos un poco más adelante, pero valga notar que a través de esta noción de lo fantasmal nos es posible entender al pasado y a la imagen que tengamos de él de una manera distinta. Por un lado, notamos que aquello que recordamos insistentemente actúa sobre nosotros y no al contrario. La memoria y el recuerdo nos sobrevienen. Por otro lado,

$7 \quad$ Ya veremos que aunque no se tenga ningún poder ni ninguna decisión sobre la invocación del fantasma - ningún poder con respecto a la determinación de la presencia del fantasma-, su presencia ejerce sobre nosotros un efecto que exige que actuemos de cierta manera. La efectividad del fantasma no se determina, entonces, por su determinación o estatus ontológico - es por definición indeterminable y ambivalente- sino por lo que exige con su presencia. En este sentido sí habría algo que se podría o que se permitiría con la presencia del fantasma. 
lo que sea esa imagen del pasado no puede pensarse como lo que nos remite a un mundo perdido, acabado, superado, sino como aquello que continúa actuando en nuestra experiencia cotidiana. La memoria no pertenece al pasado, es, ante todo, una experiencia del presente. Sin embargo, este presente ya no puede pensarse tampoco como el conjunto de lo que está actualizado y presentificado (lo idéntico a sí mismo), sino como un tiempo atravesado por el anacronismo: un presente que reúne en sí mismo otros tiempos. Es por esto que, en la medida en que la aparición del fantasma implica ya siempre una presentación de otros tiempo pasados, nos confronta necesariamente con una responsabilidad con respecto a ese pasado; su aparición señala que hay algo de ese pasado que no puede olvidarse y que merece ser recordado. En esa medida, aunque la presentación del fantasma sea intempestiva, reclama una necesidad de justicia del propio y presente tiempo: su insistencia a no desaparecer indica que es necesaria una acción en el presente que le 'haga' justicia y que asuma una responsabilidad frente a ella.

La presencia insidiosa y persistente del fantasma, dice Derrida, impone un trabajo de duelo: este es su carácter acuciante. El fantasma, en tanto que muerto-viviente que reclama una atención sobre sí, obliga a los vivos - con la fuerza de la necesidad- a 'hacer' algo con él. La noción de duelo nos permite pensar ese doble carácter involuntario y demandante de la experiencia/memoria fantasmal pues, por un lado, describe el acaecimiento de la muerte -incontrolable- y además, toda una serie de efectos, responsabilidades y decisiones que tomar, que afectan a los vivos. Si bien el acontecimiento sobreviene, sí exige de nosotros - reclama - un lugar en la memoria y ante eso no sabemos exactamente qué hacer. La muerte - ese no-suceso, la aparición de una ausencia, la des-aparición - resuena en el presente. A través del análisis del fantasma en Hamlet, Derrida nos muestra algunas maneras de realizar ese trabajo de duelo o esa forma de asumir la aparición de la desaparición o del desaparecido. En una primera instancia, parecería que lo más conveniente es, como suele decirse, 'darle un cierre al capítulo y seguir adelante'. Para esto, nada mejor que una certeza que permita conjurar de una vez por todas $-\mathrm{y}$ deshacerse de- esa presencia fasti- 
diosa. Para el trabajo de duelo, en esa dirección, "nada sería peor [...] que la confusión o la duda: es preciso saber quién está enterrado y dónde -y es preciso (saber... asegurarse de) que, en lo que queda de él, él queda ahí, ¡Que se quede ahí y no se mueva ya!” (Derrida 2012, 23). El trabajo de duelo estaría, desde este punto de vista, dirigido a asegurarse de que, en primer lugar, la pérdida llegue a ser asimilable pero esto para, en segundo lugar, asegurarse a su vez de que los muertos no vuelven a aparecer como vivos. De que los muertos estén, como dice Derrida, bien muertos y no espanten a los vivos.

Según muestra Derrida, aquellos que han sabido realizar ese trabajo de duelo por la vía de la determinación de la forma de presencia del fantasma, ya sea como una realidad o una irrealidad, pero nunca en su indeterminación y ambigüedad, son los scholars tradicionales. En efecto, el trabajo de los scholars - a los que hace referencia Derrida a partir de las palabras de Marcelo, en Hamlet, así como las perspectivas de algunos filósofos, científicos sociales, artistas, entre otros teóricos e intelectuales actuales-, sigue estando caracterizado por esa forma de duelo y esa forma de construir memoria que encuentra la única solución en el establecimiento de la verdad y de los límites entre lo real -lo que fue real- y la ficción o lo falso. El fantasma o la experiencia de lo espectral es concebida así como una situación que debe ser contrarrestada. Como un momento de transición a la verdad y a la anulación de la falsedad del pasado. Como si el fantasma se presentara para anularse a sí mismo, para que fuera, de alguna manera, exorcizado y declarado 'bien muerto' Esto, pues los scholars no han comprendido la importancia de esa forma paradójica y contradictoria de la presencia espectral:

No ha habido nunca un scholar que verdaderamente, y en tanto que tal, haya tenido nada que ver con el fantasma. Un scholar tradicional no cree en los fantasmas - ni en nada de lo que pudiera llamarse el espacio virtual de la espectralidad. No ha habido nunca un scholar que, en tanto que tal, no crea en la distinción tajante entre lo real y lo no-real, lo efectivo y lo no-efectivo, lo vivo y lo no-vivo, el ser y el no-ser [...] en la oposición entre lo que está presente y lo que no lo está, por ejemplo bajo la forma de la objetividad (Derrida 2012, 25). 
Así, el asedio de lo fantasmal, de eso del pasado que es recurrente en nuestra experiencia histórica del presente, busca ser anulado, bajo estas perspectivas, mediante la identificación de la verdad del pasado y mediante la autopsia del cadáver propio del fantasma. Lo fantasmal es así reducido a una simple fantasmagoría o ilusión que impide el acceso a la verdad histórica.

Dejemos de lado la posible crítica epistemológica y metodológica que se le podría hacer a esta perspectiva que anhela la objetividad histórica, y tratemos de ver su carácter problemático desde otro lugar: ¿Qué se pierde con la conjuración del fantasma?, ¿qué característico de esa ontología difusa y ambivalente del fantasma nos sirve para pensar posibilidades que la anulación de lo fantasmal no ofrece?, más aún, ¿a qué y para qué sirve la conjuración del fantasma, suponiendo que esa verdad sea posible de alcanzar/restituir?, ¿será que lo que nos impone semejante experiencia de asedio es simplemente la búsqueda de la verdad, o qué más podemos decir de esa forma aterradora de la memoria?

\section{La (in)determinación ontológica del fantasma: el lugar de la justicia}

La experiencia de lo fantasmal nos dice algo sobre toda forma de memoria: el recuerdo - en tanto que pasado que se hace presente- es a la vez una presencia y una no-presencia, un resto del pasado a partir del cual su presencia plena no puede ser restituida. Así pues, la memoria como el medio a partir del cual podemos decir algo acerca del pasado, nunca promete la verdad ${ }^{8}$. Por el contrario, la memoria que sobreviene y que se hace necesaria en el presente, deshace las fronteras - que desde siempre han sido difusas - entre pasado, presente y futuro. Como ya mencionábamos antes, esta memoria que se impone actualiza constantemente un resto del

8 Trataremos de ver que incluso en el reclamo por verdad de aquellas personas más directamente involucradas o afectadas por la violencia del pasado, no se trata simplemente de la verdad objetiva del scholar, sino de otro tipo de verdad que sirve a otros fines que los del conocimiento. Se trata de una verdad que no es clausura sino condición de posibilidad de otras vivencias y, más importante aún, cierta justicia. En ese sentido la memoria y el pasado dialogarían con un presente que anhela justicia - un presente que busca hablar con el fantasma y no hacerlo callar- y no un cierre - silencio, impunidad- del pasado. 
pasado pero nunca a la manera de presencia idéntica con lo real, nunca en tanto que resurrección plena del desaparecido o del mundo desaparecido (pasado, perdido). Es decir, siempre en la forma de una ausencia que vuelve. Que de esa ausencia pueda establecerse algo así como 'lo objetivo', creemos, quedan muchas dudas y problemas. Sin embargo esto nos obliga a pensar qué es aún posible en esa experiencia, lo cual, para Derrida, es lo más importante: la justicia, la responsabilidad e, incluso, el otro en su singularidad.

Derrida nos describe la reacción de Hamlet al recibir el mandato del fantasma de su padre: si bien parece que dicha reacción sigue operando bajo la lógica de la restitución de la verdad ${ }^{9}$, Hamlet reconoce que esta experiencia fantasmal, previa al contenido mismo del llamado del fantasma del padre, corresponde a una experiencia temporal dislocada. Hamlet reconoce que ese llamado por justicia solo puede surgir en un tiempo que no corresponde consigo mismo, en un tiempo out of joint: Hamlet

maldice, incluso, la suerte que le habría hecho nacer para reparar un tiempo que marcha de través. Maldice el destino que le habría destinado justamente a él, a Hamlet, a hacer justicia, a volver a poner las cosas en orden, a volver a poner la historia, el mundo, la época, el tiempo, del derecho, en el camino derecho, a fin de que, conforme a la regla de su justo funcionamiento, avance derecho $-\mathrm{y}$ según el derecho- (Derrida 2012, 34).

La respuesta de Hamlet ante la aparición del fantasma de su padre es, en primer lugar, de desasosiego y apremio pues comprende que un tiempo en el que los fantasmas son posibles, es un tiempo dislocado, fuera de quicio, desfasado con respecto a sí mismo. Es decir, un tiempo en el cual 'lo real' no corresponde consigo mismo pues hay presencias ('irrealidades') de un pasado que 'debería' haber desaparecido. Se trata, en otras palabras, de la experiencia de un pasado que no ha pasado sino que está presente - tal

9 Ya veremos que la diferencia entre estas dos formas de responder al fantasma que estamos a punto de describir es análoga — tal vez sea igual — a la diferencia entre la justicia entendida como la aplicación de la ley y el ejercicio del derecho, y otra forma de justicia que excede los límites justos del derecho. 
como el fantasma- y que con ello indica que el tiempo está fuera de $s u$ lugar y que hay algo que no anda bien. No obstante y más allá de que Hamlet piense que su tarea consiste en poner el tiempo al derecho y con ello vengar el asesinato de su padre ${ }^{10}$, es de suma importancia notar acá que el llamado de justicia está precedido y condicionado precisamente por esa experiencia temporal desfasada. Solo puede construirse 'justicia' - $\mathrm{o}$ solo se puede pretender construirla - en un tiempo en el cual las cosas no van bien - señalando un tiempo fuera de sí, desquiciado- y en un tiempo en el cual el pasado asedia a los vivos. Sin el asedio, no hay llamado, y sin el llamado, no es posible anhelar justicia. El desajuste temporal es entonces condición de posibilidad de la justicia; la violencia con que se impone el pasado - tal como lo vivimos y experimentamos hoy, por ejemplo, en nuestra localización histórica- no es la indicación de que la verdad sea necesaria para superar el pasado, sino de que es el momento en el que hacer justicia es necesario y urgente.

¿Cómo entender entonces esta justicia?, ¿cómo y de qué manera podemos responder a esta situación de apremio histórico? Si no podemos entender el duelo como lo que restituye el cadáver del muerto -resucitarlo, y con ello anular su condición espectral- entonces, la justicia que se deba hacer con él, responde - habla - al fantasma en tanto que tal. Se trataría de una forma de reparar el tiempo que no es equiparable al de la restitución del pasado ni al ajustamiento del pasado a un presente idéntico a sí mismo. Sin embargo, esto trae una serie de problemas y cuestiones por pensar, entre esas, que "se pasa fácilmente de lo desajustado a lo injusto" (Derrida 2012, 33), pues habitualmente se ha pensado que lo que desajusta el orden y lo que se impone con violencia — más aún, lo que aparece violentamente-, es de hecho lo injusto y lo que merece y debe ser puesto al derecho. Esta última perspectiva es característica del derecho y de su noción de justicia.

En su texto Fuerza de ley, Derrida somete a crítica —en clave benjaminiana - la distinción entre la violencia legítima y la ilegítima que está a la base del derecho. Tal como muestra Walter Benjamin en Para una

10 Como si hacer justicia correspondiera precisamente con esto: volver a hacer al tiempo idéntico a sí mismo, vengar el pasado para que su sentido en el presente quede clausurado y se alcance paz. 
crítica de la violencia, Derrida afirma que no hay diferencia sustancial entre la violencia legítima y la violencia del delincuente y del injusto. La fuerza con la que la ley se impondría sobre sujetos y ciudadanos, estaría justificada solo en tanto que instituyente del orden legal legítimo. La violencia con la que la ley se impone no es justificada por sus fines ni por su naturaleza, sino simplemente en tanto que es fundamento del derecho mismo. Su legitimidad está dada por su propia fuerza y por su capacidad de mantener un orden ajustado. En otras palabras, es legítima la violencia que permite que las cosas estén en $s u$ lugar, ajustadas a $s u$ lugar.

No obstante, esta forma de hacer justicia, a través de la violencia que ajusta, no se limita simplemente al orden jurídico ni al derecho. La ley puede ser "directa o no, física o simbólica, exterior o interior, brutal o sutilmente discursiva - o incluso hermenéutica-, coercitiva o regulativa, etc." (Derrida 1997, 17). En términos generales, se trata de la justicia como necesidad de cumplir con un deber "porque se me ha impuesto como una suerte de obligación o de condición, por medio de una especie de fuerza simbólica o de ley, en una situación que no controlo" (Derrida 1997, 17). La ley - la costumbre, lo habituado, las formas recurrentes y justas (o 'precisas') de hablar de algo y de actuar- codifica el horizonte de lo que es justo y segrega, por defecto, a toda otra forma de violencia como ilegítima y por lo tanto injusta. Una vez disuelta la dicotomía violencia legal/ ilegal, lo que se manifiesta es que el ajuste que la ley - lo que configura el orden 'justo' o 'ajustado'- hace del tiempo desajustado, no es más justa con el pasado, sino tan solo legitimada en tanto que la violencia que ejerce con el pasado es la ajustada o la precisa, en el sentido de la que corresponde a lo que 'se debe' o a lo 'legal'. Más aún, esta violencia inherente a todo orden legal oculta su carácter violento en tanto que se muestra como necesaria para la constitución de un orden regulado y armónico. Este ocultamiento de la violencia constitutiva de lo legal se expresa en la forma de la conjuración del fantasma, puesto que anula su diferencia y desajuste del tiempo, traduciéndolo e incorporándolo al orden de lo ajustado. El pasado fantasmal pierde entonces su potencia y el out of joint es anulado. La consecuencia de esto es, al imposibilitar la prolongación del tiempo espectral, la obstaculización del reclamo por otra forma de justicia que surge de otro ajuste del tiempo. 
La justicia que pretende el derecho - que las cosas estén al derechoclausura un espacio abierto por la aparición del espectro. Una vez que el derecho actúa, las verdades se piensan como determinadas, los sujetos identificados - las identidades definidas- y las acciones encuentran su sentido último. El fantasma se exorciza y se declara la verdad de su muerte; desparece por completo para dar lugar al hecho objetivo: la determinación de una presencia plena en el pasado que ahora se constituye como verdad histórica, imposibilita que esa ausencia tenga más una presencia. Con esto no se consigue la determinación o construcción de una memoria justa, sino, por el contrario, el cierre del sentido y la pretendida superación del evento. Esta determinación cumple la función de permitir olvidar y que el pasado no vuelva más a aparecer y asediar. Sin embargo, si asumimos que la condición de la justicia es el desajuste del propio tiempo, entonces habría que pensar una forma de justicia que escapa y excede a la justicia que permite y protege el derecho.

\section{El caso de la masacre de Trujillo: ¿qué lugar tienen los fantasmas?}

La posibilidad de la construcción de memoria está dada porque su carácter fantasmal no es nunca anulado. La memoria es siempre memoria fantasmal: memoria que se hace actual y que reclama presencia insistentemente. Lo superado, acabado y dejado atrás, es olvidado y no recordado. Como ya lo mencionábamos más atrás, la posibilidad de otra forma de justicia está dada por el poder dirigir la palabra al fantasma en tanto que tal. El objetivo no sería acabar con el fantasma y su sentido, sino hacer algo con él ${ }^{11}$.

Ahora bien, ¿cómo hacer algo con el fantasma si ya no se trata de hallar su verdad?, ¿qué hacer con él si la cuestión no es volver a ajustar

11 Vale la pena aclarar que esto que se haga con el fantasma no tiene que ver con una especie de acción intencional. Se trata de lo contrario: de cómo el pasado pasma, asedia y obliga a ser recordado, y posteriormente, obliga así mismo a actuar. Tampoco tiene que ver con que se haga algo con el fantasma pues esto no implica que se haga de él/ello algo determinado en el presente. Se trata, más bien, de hacer algo de él en tanto que nos obliga a hacer algo con otro y que nunca nos dejará de imponer tareas y obligaciones. El fantasma está con nosotros para toda la vida, pues lo justo nunca termina de llegar. 
el tiempo?, más aún, ¿cómo pensar que sea posible hacerle justicia al pasado si la memoria es siempre desajustada? No se trata, como se podría pensar, de anular la violencia con la que se impone el pasado ni la violencia que se podría ejercer en tratar de 'reparar' ese tiempo; por el contrario, consideramos que hay que asumir y acoger esa violencia. En efecto, en primer lugar, la situación de urgencia y asedio que impone el fantasma, su carácter involuntario y por tanto coercitivo, no puede seguir pensándose como una situación que debe ser contrarrestada; como si fuera posible un momento de paz plena en donde los espectros no fueran ya posibles. Esto nos remite al segundo punto, a saber, que la manera en que respondamos ante la aparición del fantasma - nuestra responsabilidad - nunca va a poder ajustarse plenamente al fantasma, al otro - como si tuviera una presencia, una forma de ser plena reconocible ${ }^{12}-$ y por ello se trata de responder no al muerto-viviente ni a su verdad, sino a su ausencia. En esta medida, nos dice Derrida, la tarea de hacerle justicia al pasado no puede ser pensada como la restitución y el reemplazo de la presencia por la ausencia - no es la lucha por el reconocimiento de la verdad, o del otro-, sino lo que se puede lograr y producir en ese intervalo entre 'lo que es' y aquello que lo desacomoda y desajusta: "Mantener unido lo que no se mantiene unido, y la disparidad misma, la misma disparidad [...] es algo que sólo puede ser pensado en un tiempo de presente dislocado, en la juntura de un tiempo radicalmente dis-yunto, sin conjunción asegurada" (Derrida 2012, 31). Se trata de una forma de justicia que no anhela la afirmación de lo mismo sino que está posibilitada por una diferencia y un constante diferir del

12 A este respecto nos parece sugestiva la asociación - que el mismo Derrida hace- entre la noción de fantasma y la de huella. Si bien uno podría pensar que el valor y el significado de una huella están dados por la posibilidad de remitir a algo más —a un animal, a una persona, o sea lo que sea que la haya marcado-, la huella en sí misma expresa una ausencia. Expresa que algo pasó por allí, no se sabe muy bien cuándo, quién, ni en qué circunstancias, pero que pasó y ha dejado una marca que interpretamos en el presente. Sin embargo, lo interesante de esta noción es que, a pesar de las incertidumbres y las posibles equivocaciones que podamos cometer al darle un sentido a la huella, ella está allí para ser interpretada y para que nosotros le demos un sentido. No hacerlo sería reducirla a una insignificancia y, con ello, no solo el 'hecho' quedaría olvidado, sino la misma ausencia quedaría invisible. Se trata, entonces, de hacer que la ausencia tenga un lugar. 
pasado. Esta especie de 'impropiedad' del fantasma implica que todo aquello que nos atrevamos a decir de él, está sometido a un siempre e inevitable desajuste.

A este último respecto consideramos interesante el trabajo que realiza la Asociación de Familiares de Víctimas de Trujillo (AFAVIT). Su insistencia por recordar a los desaparecidos en la masacre de Trujillo (Valle del Cauca) y en otros hechos atroces en este mismo departamento a manos de diversos actores armados (legales e ilegales), nos muestra que, sin duda, el reconocimiento estatal, sus medidas de protección, apoyo a las víctimas y juicio de los victimarios, son importantes, pero que hay otros asuntos y preocupaciones que son necesarios resaltar y que siguen concerniendo a una búsqueda por 'hacer' justicia. En esa medida consideramos que el caso de la AFAVIT nos enseña una importante lección acerca de lo que está en juego con los ejercicios de construcción de memoria histórica, pues muestra que estos deben atender también a estas otras preocupaciones y que nosotros desde la academia, desde las ciencias sociales y el derecho, no podemos perder de vista que el sentido de justicia no puede reducirse a lo legal o a lo posibilitado por la ley.

En el terreno jurídico, la AFAVIT ha heredado la voz del reclamo de justicia de los padres jesuitas Javier Giraldo y Tiberio Fernández, quienes desde 1988 emprendieron trabajos de recolección de datos y testimonios. El movimiento eclesial llevó el caso de Trujillo ante la Comisión Interamericana de Derechos Humanos (CIDH) en 1995 cuando el Estado colombiano, mediante "dos sentencias absolutorias, negaron el único derecho de los familiares de las víctimas: el castigo a los culpables y el repudio de una sociedad a la destrucción de la vida humana" (AFAVIT 2015). El 31 de enero de 1995, el Estado colombiano reconoció su responsabilidad en la masacre ante el organismo internacional y posteriormente a dicho reconocimiento nace la AFAVIT, la cual ha estado desde entonces al tanto de las sentencias y los pronunciamientos del Estado colombiano concernientes a la masacre de Trujillo; ha denunciado la inoperancia del Estado y se ha resistido a permitir que el caso quede en la impunidad. Estas acciones demuestran que la AFAVIT no está desentendida de la necesidad de una justicia legal y que de hecho anhela que la verdad de los hechos pueda ser determinada 
así como los responsables y las medidas legales correspondientes. Sin embargo, la Asociación ha asumido su labor como una que va más allá de una determinación de los hechos del pasado y del reconocimiento de las identidades de las víctimas y victimarios. Ellos afirman que "a la masacre física de vidas humanas, siguió una segunda masacre: la masacre moral. Las autoridades llamadas a impartir justicia, desconocieron los testimonios y pruebas aportados por testigos presenciales, que a la postre fueron también víctimas de los sicarios" (AFAVIT 2015). Es decir, la AFAVIT asume un compromiso, a través de la memoria, con el presente y el futuro: su duelo no termina en la verdad, no hay, en el sentido estricto de la palabra, reconciliación absoluta con el pasado, pues este continúa teniendo un lugar en su comunidad.

Es de vital importancia notar aquí lo que la AFAVIT llama 'segunda masacre' y que asocia a un daño moral. Si la labor de la Asociación estuviera reducida al esclarecimiento de la 'verdad histórica', su labor podría ser fácilmente delegada a los medios estatales, a sus representantes en el gobierno, o a la sociología y la ciencia política. Sin embargo, la AFAVIT asume la responsabilidad de velar porque también se haga justicia ante esa segunda masacre: la masacre del acallamiento de los vivos que reclaman que se siga haciendo justicia y se continúe construyendo memoria; la injusticia en contra de los vivos que siguen sufriendo y temiendo por sus propias vidas; la violencia que resuena en la vida cotidiana y en la memoria de los habitantes de Trujillo. Ante estas injusticias, el Estado ha intentado y prometido asumir una labor de protección y prevención, pero esta no ha mermado la profunda sensación de injusticia en la comunidad - el 'daño moral'-, ni ha conllevado a la protección efectiva de sus líderes comunitarios. A pesar de esto y aunque parezca que nadie puede reconocer ni responder a ese reclamo de justicia por esta otra masacre, estas personas sienten aún la urgencia y necesidad de seguir recordando, a pesar de los riesgos y peligros que esto les ha acarreado. Es por ello que en este caso el trabajo de memoria no se reduce al esclarecimiento de la verdad, sino a un continuo reclamo de justicia que halla su potencia de acción en las injusticias del pasado. Son los fantasmas de la masacre, esas personas que permanecen en la memoria de sus familiares, quienes los impulsan a no desfallecer en el 
anhelo de justicia. Es por ellos que sus familiares asumen una tarea ética y política dentro de su comunidad.

Luego de la denuncia de la negligencia del Estado colombiano, la CIDH publicó un informe en el cual responsabilizaba al Estado colombiano por las muertes y los hechos violentos ocurridos entre 1986 y $1994^{13}$. El gobierno de ese entonces, liderado por Ernesto Samper Pizano, aceptó las recomendaciones y las inculpaciones de la $\mathrm{CIDH}$ y se comprometió a reparar a las víctimas. El presidente en ese momento dijo: "Espero finalmente que nuestros hijos algún día nos perdonen, cuando estén respirando el aire puro de la paz, el haber sentido tanto odio cuando teníamos tanto derecho a la esperanza"(AFAVIT 2015). Sin embargo, ese aire de paz está lejos de ser 'puro'. Por el contrario, la dificultad reside en tratar de volver a encontrar paz en un tiempo tan impuro y tan atravesado por la injusticia. Una de las medidas tomadas para reparar los daños morales sufridos por la población de Trujillo, fue la de construir un Parque Monumento que "rinde homenaje a las víctimas de un género de violencia que, de silenciarlo y olvidarlo, ese silencio y ese olvido serían destructivos de lo que protege a la especie humana como tal"(AFAVIT 2015). El Parque Monumento no es la re-construcción de un lugar devastado por la violencia, sino un recuerdo viviente de esa violencia. El dolor y la muerte no están allí para ser negados, sino para que tengan siempre un lugar. El aire puro de la paz no circulará nunca por Trujillo porque la justicia que tanto reclaman las víctimas y familiares de las víctimas es incompatible con él: su justicia solo puede tener lugar cuando las ausencias siguen presentes y cuando no se ha negado la violencia que ha pasado por allí. Al respecto la AFAVIT ha publicado este poema que habla sobre aquel Parque Monumento:

¿Es lugar de muertos o de vivos? ¡Se pregunta hoy asombrado!

De los muertos hay siembra de raíces, símbolo de memoria, de los muertos nacen flores, expresión de resistencia,

13 La violencia en la región de Trujillo no ha cesado aún. No solo su presencia en la cotidianeidad de la población lo demuestra, sino otros crímenes cometidos en contra de personas relacionadas a la Asociación. Ver: http://www.afavit.com/comunicados/Alba\%20Chelito. html. Consultado el 2 de marzo de 2015. 
de los muertos salen rostros que hablan de proyectos.

Es la vida que trasciende más allá de la muerte, es el Parque-Monumento, reparación, dignidad, es espacio de justicia, lucha contra la impunidad, no es lugar de muertos, es lugar de vivos gritando libertad.

Los muertos aparecen vivos en cuerpos esculpidos, son los huesos secos que se unen en grito de justicia,

los muertos se levantan, nos miran y nos hablan,

la siembra de semillas convertida en esperanza.

No es lugar de muertos, es jardín de vivos, olor a resistencia, es memoria histórica, denuncia subversiva, es duelo doloroso de llanto y sufrimiento, es sueño y utopía de nueva sociedad (AFAVIT 2015).

El Parque Monumento, como lugar en el cual se construye la historia y la memoria de Trujillo, no respira aire de paz ni de tranquilidad. Es un lugar habitado por los muertos, un lugar donde los fantasmas están con los vivos. Pero esto no significa que sea un lugar para la eterna lamentación ni para el desconsuelo. Por el contrario, es el lugar donde se potencia la lucha por la justicia. Es su relación anacrónica con el pasado - su estar con los muertos- lo que les posibilita asumir una posición de lucha en el presente. Nos atreveríamos a decir que los habitantes de Trujillo están con sus fantasmas en ese lugar. El pasado y su ausencia tienen lugar allí.

Mas ¿es esta relación con el pasado un caso extremadamente particular? Tal vez lo sea, pero resulta significativo que en la experiencia de esta comunidad la pregunta por el pasado no es una pregunta que se limite por la verdad o por el conocimiento, sino que concierne a la vida misma, a la existencia, a la esperanza, a la justicia. Y esto resulta significativo pues es ese vínculo anacrónico entre el pasado y el presente - el vínculo que hace preciso (urgente) a un pasado en un presente- el que debemos rescatar $\mathrm{y}$ tener en mente en la manera en que construimos historia y pensamos nuestro pasado. Pues una historia que no demuestra en qué medida su labor contribuye al presente, no hace verdaderamente memoria ni permite justicia. No se trata de estar en paz con el pasado y que tras los monu- 
mentos quede sepultado, se trata de que el monumento sea un memorial, un lugar para la memoria y para que los desaparecidos estén.

Para apreciar la singularidad de la labor asumida por la AFAVIT, creemos que vale la pena explorar una perspectiva un poco distinta: la labor de los últimos años del Centro Nacional de Memoria Histórica $(\mathrm{CNMH})$ que ha hecho un trabajo exhaustivo de análisis y recolección de datos con el fin de

[c]ontribuir a la realización de la reparación integral y el derecho a la verdad del que son titulares las víctimas y la sociedad en su conjunto así como al deber de memoria del Estado con ocasión de las violaciones ocurridas en el marco del conflicto armado colombiano, en un horizonte de construcción de paz, democratización y reconciliación (Centro Nacional de Memoria Histórica).

Uno de los informes del CNMH está concentrado en la masacre de Trujillo y una de sus secciones está dedicada a dar

un lugar a las voces y memorias de las víctimas sobrevivientes y los familiares de los desaparecidos, torturados y asesinados durante la masacre, y demostrar cómo los narcotraficantes, en asocio con militares y grupos paramilitares, por más poder económico, político y militar que controlen, no pudieron ni han podido hasta ahora imponer su interpretación de los hechos en el campo de las memorias sociales (Centro Nacional de Memoria Histórica 2008, 200).

Si bien el CNMH reconoce la importancia del trabajo de organizaciones como la AFAVIT, admite también que la "reparación integral" ${ }^{14}$ debe pasar por unas políticas de restitución de tierras y de reparación que requieren "el compromiso y la voluntad política del

14 Y con reparación integral el CNMH entiende "una que reconozca a las víctimas como portadoras de derechos, ciudadanos que reclaman no solo por aspectos económicos, sino también simbólicos que les devuelvan la dignidad y la singularidad humana a las victimas. En este sentido, la reparación integral pasa por políticas redistributivas y por políticas de verdad y justicia" (Centro Nacional de Memoria Histórica 2008, 201). 
Estado" (Centro Nacional de Memoria Histórica 2008, 201). Como ya vimos, para Derrida - siguiendo a Benjamin - hay una dificultad intrínseca al lenguaje del Estado y de la ley en la medida en que niega su propio proceder violento en el ajuste de un tiempo desesperanzado a un tiempo armónico y 'justo'. Mas creo que vale la pena preguntarnos por el tipo de justicia que estaría permitida por ese compromiso y esa voluntad del Estado y, más aún, cuál es el lugar que según esta perspectiva tendrían los fantasmas en la 'reparación integral' de las víctimas del conflicto colombiano.

El informe del CNMH titulado Trujillo: Una tragedia que no cesa, en la segunda sección se ocupa de los trabajos de memoria histórica asumidos por la comunidad y resalta el trabajo de la AFAVIT. Sin embargo, el CNMH parece preocupado por el enfoque de las actividades y conmemoraciones realizadas por la, que ellos llaman, organización más reconocida por la comunidad de Trujillo; esto dado que, para ellos, existe el riesgo de que

las actividades conmemorativas y de elaboración del duelo de las victimas y AFAVIT se queden en la conmemoración y ritualización del pasado, [y] les deje suspendidos en el tiempo y obstaculice tanto la reconstrucción de sus vidas como su proyección al ámbito público, eficacia política y habilidad para comunicar a otros sus memorias, verdades y aprendizajes (Centro Nacional de Memoria Histórica 2008, 203).

Si bien es cierto que un anclaje en el pasado no puede ser sino perjudicial para la vida, el CNMH reprocha esa 'suspensión en el tiempo' ya que puede perjudicar la 'eficacia' política y comunicativa de la organización. Sin embargo, como vimos un poco más atrás, esa 'suspensión' en el tiempo, no implica necesariamente anular la participación política y social de la organización, sino que más bien la ha impulsado de diferentes maneras, a través de un sinnúmero de prácticas y de discursos de diversa naturaleza. En ese sentido, las palabras del CNMH parecen indicar que se evalúa la efectividad política y comunicativa en otro sentido al que asume la propia organización. Es decir, asume lo 'efectivo' y lo preciso en tanto que funciona, sirve o se adecúa al plan de reparación estatal. 
Llegados a este punto, podemos ver una diferencia importante entre lo que la AFAVIT piensa de su relación con el pasado y lo que parece que espera el Estado de ellos. Parece ser que a partir de la perspectiva de la 'reparación integral', el objetivo es 'devolverle' a las víctimas y sus familiares algo que habían perdido, todo esto a través de una reparación construida por la 'verdad'. El lugar del fantasma en esta perspectiva es el de la inminente desaparición puesto que su lugar debe ser reemplazado por el de 'la verdad' para dar lugar a la 'restitución, a la 'devolución', al 'aire de paz'. Una vez el fantasma ha aparecido, ya no puede desaparecer ni no-ser absolutamente. Y es que su aparición señala también una pérdida irreparable: que haya fantasmas implica que el tiempo está inevitablemente desajustado y no hay nada que podamos hacer para volver a ajustarlo. No podemos devolver el tiempo. Sin embargo, el trabajo de memoria de la AFAVIT consiste en, precisamente, actuar en ese tiempo suspendido, en ese presente atravesado de principio a fin por los fantasmas y los espectros. Tal como lo afirmó uno de los participantes de los talleres realizados por el CNMH en el 2008: “Todos los días recordamos porque nuestras vidas cambiaron por ello [...] la violencia fue una marca. No se puede olvidar" (Centro Nacional de Memoria Histórica 2008, 229). El duelo se hace con los muertos y no a pesar de ellos: he ahí la dificultad de todo trabajo de duelo.

No obstante, resulta interesante y sugestivo que trabajos como el del $\mathrm{CNMH}$ hagan énfasis en la necesidad del olvido y nos invite a pensar en algo que tal vez quedaba sugerido más atrás, a saber, que dada la naturaleza 'parcial' de todo trabajo de memoria, ninguna narrativa o forma de memoria se opone al olvido. Por el contrario, toda narración se encuentra atravesada por olvidos, énfasis e intereses particulares que configuran la decisión de relatar la historia de una manera determinada. Es por esto que la actualización del fantasma siempre se da de formas diversas y diferentes; por lo tanto, no todo trabajo de memoria histórica tiene que ser un trabajo de remisión y reconstrucción de los hechos del pasado. En el caso de Trujillo y de las actividades realizadas por la AFAVIT, es claro que a veces solo hace falta hacer algo en nombre de los desaparecidos para hacerle honra a su ausencia. Esto es decir que en las ruinas de la catástrofe y de la muerte, la nueva vida pueda surgir; esa 
nueva vida, que de cualquier manera, siempre estará relacionada con la catástrofe y en cada aspecto de la nueva vida el pasado espectral estará presente y algunas veces podrá hacerse casi presente. Así, la responsabilidad de la memoria histórica se encuentra en este aspecto en el trabajo y las acciones que se realizan en las ruinas de la catástrofe.

En esa medida, podría decirse entonces que aquellos que asumen esta tarea de redención y de lucha, de acción productora de memoria en circunstancias de adversidad y de peligro, son de hecho los herederos de los fantasmas. Y el que no quiere tener nada que ver con el fantasma no lo hereda, lo niega, lo hace desaparecer por completo, contribuye a la impunidad. Es por esto que el modo de estar-con los fantasmas en este sitio, invita a acciones, proyectos y voces de reivindicación. En efecto, la AFAVIT afirma que: "Rescatar su memoria es reivindicar la posibilidad de ser humanos y de conjurar las fuerzas que amenazan con destruir lo elementalmente humano" (Centro Nacional de Memoria Histórica $2008,229)$. De esta forma, podemos ver que solo a través de esa relación con el pasado, la reivindicación, la esperanza y la vida son posibles. Es por esto que su labor de memoria no solo cobra la forma de narrativa histórica, sino además la forma de talleres, marchas, diálogos y peregrinaciones que permiten construir una comunidad no a pesar de los fantasmas, sino en nombre de ellos. Este es el homenaje que a través de la memoria le rinden a los fantasmas: la construcción de nueva vida ${ }^{15}$.

Resulta interesante que Derrida aborda la noción de herencia y afirma que ser heredero de un muerto no consiste en ser semejante al fantasma - parecerse a él-o en saber quién era, y por ello estar capaci-

15 A este respecto el informe de Centro Nacional de Memoria Histórica ofrece múltiples testimonios de víctimas y familiares de víctimas que encuentran en su relación con el pasado, la posibilidad y la potencia para seguir con su vida, transformarla. Un ejemplo de ello es el testimonio de Yamileth Vargas del 2004 cuando esculpieron los osarios que están en el Parque Monumento: "Rehacer las esculturas de los osarios nos ha permitido reencontrarnos con nuestros seres queridos. Volver a acariciarlos, hablarles y sentirlos, nos ha dado fortaleza, ganas de seguir luchando por nuestro derecho a la justicia, a mantener viva la memoria... su vida se reivindica con cada escultura, y su amor y memoria viven en nuestros corazones". (CNMH 2008, 238).

"Por esta masacre vivimos la vida cada año, con las peregrinaciones, con las visitas, con los lazos y hermandades nacionales e internacionales, y eso es esperanza, eso es lo que nos salva". (Maritze Trigos) (CNMH Trujillo, 238). 
tado para decir su verdad, sino de quién se atreve, en el desajuste de toda relación entre los vivos y los muertos, a honrar al muerto. Para ello "es preciso ${ }^{16}$ filtrar, cribar, criticar, hay que escoger entre los varios posibles que habitaban la misma inyunción [...] si la legibilidad de un legado fuera dada, natural, transparente, unívoca, si no apelara y al mismo tiempo desafiara a la interpretación, aquél nunca podría ser heredado" (Derrida 2012, 30). El fantasma asedia e impone, y la tarea de los vivos es hacer de su legado, de la indicación de su ausencia, un don. La aparición violenta del fantasma impone una necesidad: hacer algo con él, interpretar su herencia, su ausencia, y tomar una decisión en el presente. El pasado no asedia a los vivos para que estos se limiten a preocuparse por recolectar datos, evidencias, información, etc., y que con ello la imagen del otro sea más 'verídica', sino para que lo transformen en algo asimilable y significativo en su presente. Una vez más insistimos en que el caso de la AFAVIT nos enseña y nos pone de manifiesto este punto. En su relación con el pasado la verdad no es un dato sin más, sino que es una perspectiva que posibilita formas de vida y que permite asumir ciertas posiciones existenciales. La academia no puede entonces desentenderse de los objetivos ni de las consecuencias de sus contribuciones a la comprensión del pasado violento colombiano, ni mucho menos desentenderse de las responsabilidades que se asumen en esta labor teórica e interpretativa del presente colombiano. Más aún, solo una vez vinculada la construcción de memoria con nuestras vidas, es que 'realmente' estaremos contribuyendo a la construcción de justicia. Es por esto que el hecho de que el pasado sea necesario para el presente indica la necesidad propia de un presente. Para Derrida, responder a esta necesidad constituye la justicia. La justicia es por lo tanto un asunto de necesidad y de responder a lo que se impone con violencia. Hagamos entonces que nuestra labor como científicos sociales y teóricos de nuestra realidad nos imponga una necesidad de acción y una necesidad de transformar nuestra propia vida y nuestra propia comunidad. Un fantasma a la distancia no es un fantasma, es un cadáver. Cuando

16 Nótese que lo que es preciso no es 'lo preciso', sino lo que es necesario hacer en una situación enigmática. 
podamos estar con ellos, podremos hacer algo con ellos en el anhelo de una justicia venidera y de una vida por-venir.

\section{Conclusiones}

Si bien se podría pensar que esta escogencia del pasado, esta decisión de hacer del fantasma uno determinado - escoger heredarlo de determinada manera-, sería cometer una cierta interpretación parcial y por ello una injusticia, debemos recordar que el pasado siempre se presenta desajustado. La manera en que podamos 'repararlo' consiste, más bien, en una justicia que nunca es, propiamente hablando, plenamente realizada: "se trata de donar. De donar la Diké. No de hacer justicia, de traerla de vuelta según el castigo, pago o la expiación, como se traduce la mayoría de las veces [...] Se trata, en primer lugar, de un don sin restitución, sin cálculo, sin contabilidad" (Derrida 2012, 39). Se trata de una respuesta al fantasma en tanto que tal, y por ello, no justa ni adecuada al tiempo pasado. Se trata de una respuesta que, como hemos visto, es indisociable e inseparable de su necesidad actual. La respuesta, entonces, se hace precisa y justa en la medida en que se hace acuciante, pues la herencia otorgada y traída por el fantasma no es casual ni accidental, sino auténticamente necesaria y apela a todo lo que somos. "Somos herederos, eso no quiere decir que tengamos o que recibamos esto o aquello, que tal herencia nos enriquezca un día con esto o con aquello, sino que el ser de lo que somos es, ante todo, herencia, lo queramos y lo sepamos o no [...] Testimoniar sería testimoniar lo que somos en tanto que heredamos de ello, y he ahí el círculo, he ahí la suerte o la finitud, heredamos aquello mismo que nos permite testimoniar de ello" (Derrida 2012, 68). El fantasma que asedia nos habla a nosotros en tanto que herederos de un pasado y en tanto que elegidos ${ }^{17}$ para 'reparar' la época. Responder a ese llamado del pasado es, por lo tanto, responder ante una situación acuciante del presente. Responder al llamado del pasado es, así, tomar responsabilidad en el presente.

17 Walter Benjamin habla en sus tesis de la historia en Sobre el concepto de historia, de que "hemos sido esperados en la tierra". Pero que nosotros, aquí para Benjamin como para Derrida, seamos 'los esperados', tiene que ver con que la justicia del presente encuentra su fuerza y potencia en la redención de un pasado espectral. 
Es por esto que la construcción de historia y memoria no puede concebirse como lo que hace justicia al pasado en tanto que encuentra $s u$ sentido propio y por ello imposibilita la nueva venida del espectro. La memoria tiene que dejar lugar para que la nueva venida pueda suceder. Sin ese resquicio para que el fantasma vuelva a aparecer y así desajustar de nuevo el tiempo, no queda esperanza para el pasado ni para el presente: la justicia es indisociable del por-venir indeterminable del fantasma. Ninguna esperanza ni ninguna necesidad de hacer justicia se presenta en un tiempo armónico, idéntico o posiblemente idéntico a sí. Ningún 'aire de paz' se puede respirar en el tiempo de los fantasmas, solo la confrontación y el conflicto puede surgir aquí. Pero puede que esto mismo pueda llamarse 'paz': la infinita posibilidad de disentir, de discutir, sin que ninguna voz aniquile o haga desaparecer a ninguna otra voz.

La esperanza anhela la transformación y solo puede anhelarse algún tipo de justicia en tanto que la respuesta que demos al fantasma se atreva a hacerlo - transformarlo- interpretando injustamente -en un tiempo que no es justo- a ese pasado. Por paradójico que parezca, ningún tipo de justicia será posible si no se desajusta el propio tiempo para que se dé una nueva y propia necesidad. Es por esto que la construcción actual de memoria debe preguntarse por el tipo de respuesta que se le está dando al pasado y si esta, de alguna manera, indica realmente una herencia justa y necesaria. Las diversas perspectivas en la construcción actual de memoria deben preguntarse por cómo la manera en que se hereda a estos otros fantasmales, tiene que ver directamente con la manera en que nos pensamos a nosotros mismos y los otros en nuestra convivencia en el presente y en el mundo. Ya no puede pensarse más que el pasado no esté implicado en nuestro presente, sino, por el contrario, que no es posible un presente justo sin un pasado que lo desajuste y le imponga tareas y necesidades. Nuestros fantasmas nos enseñan que nuestro propio tiempo está desajustado, pero no podemos reducir su insistencia y su presencia a la adecuación y reformación de este tiempo, sino que debemos hacerle justicia a ese desajuste: hacerlo visible, hacerlo presente - siempre-presente, siempre-por venir, que la ausencia siempre pueda volver - para que con ello alguna justicia con el otro pueda tener lugar, para que el mundo pueda ser, justamente en la revelación de su desajuste, otro. 


\section{Bibliografía}

AFAVIT. 2015. http://www.afavit.com/index.html, consultado el 5 de marzo de 2015.

Benjamin, Walter. 2000. “Sobre el concepto de historia” En Dialéctica en suspenso: Fragmentos sobre la historia. Traducción, introducción y notas de Pablo Oyarzún Robles. Santiago: LOM Ediciones.

Centro Nacional de Memoria Histórica. 2008. Trujillo: Una tragedia que no cesa. Bogotá: Taurus.

Derrida, Jacques. 2012. Espectros de Marx: El estado de la deuda, el trabajo del duelo y la nueva Internacional. Traducción de José Miguel Alarcón y Cristina de Peretti. Madrid: Editorial Trotta, 5ta Edición.

Derrida, Jacques. Fuerza de Ley: el fundamento místico de la autoridad. 1997. Traducción de Adolfo Barberá y Patricio Peñalver Gómez. Madrid: Tecnos. 